no urgent need for operation, ordered atropine 1 per cent. with cocaine 2 per cent. to be instilled and hot fomentations to be applied every three hours. The right eye was quiet; pupil active to light ; fundus normal; vision good.

October 13th. The pupil was still firmly adherent and tension had become raised a little. Under cocaine and adrenalin anaesthesia I washed out the conjunctival sac with sterile merculy perchloride solution 1 in 5,000 and mercury perchloride solution 1 in 5,000 and performed a paracentesis of the anterior chamber, removing some of the pas to a culture tube of

continued as before.

Bacteriological Report.-“"Culture on Buchanan's medium shows a pure growth of an organism morphologically identical with the meningococcus.'

The next day the pupil started to dilate, and on October 15th had given for three-quarters of the circumference. On October 16th it was quite free and the anterior chamber was clearing; a thick ring of yellow exudate was left on the anterior capsule of the lens. The tension was about normal and vision perception of light. The facial paralysis had practically recovered.

The eye continued to clear under local and general treatment, and on October 20th the pupil was well dilated, episcleral swelling subsided; tension was abont equal in both eyes, and both subnormal. Vision and both subnormal. details could be made out, and only a yellow-green reflex obtained. On convergence the left eye already diverged. The local treatment was now dionine 0.0005 gram, atropine 1 per cent. every six hours, and fomentations as before. A culture
taken from the conjunctival sac showed a growth of Stapfiylotaken from the conjunctival sac sho

On October 27 th the eye was quieter; the pupil was dilated, On October 27th the eye was quieter; the pupil was dilated,
with one small adhesion at ten o'clock. The anterior chamber with one small adhesion at ten o'clock. The anterior chamber
was clear, the lens capsule nearly clear, the vitreous clearing ; only greenish-grey reflex and gross floating opacities were made out. Vision was perception of light, and becoming worse. Atropine and dionine now only bare dilatation.

On November lst the eye was quiet, the iris showed several snail-track-like grey eroded patches scattered around, the anterior chamber was clear and of medium depth, anterior part of vitreous fluid and clear, and a large yellow-green mass was seen floating in upper quadrant; neither vessels nor disc could be geen. The tencion was - l and the vision perception of light be geen. The and the eye was apparently beginning to shrink. The general treatment has been to draw off 15 to $20 \mathrm{c.cm}$. of cerebro-spinal fluid from the lumbar region and replace with an equal amount of antimeningococcus serum about every two days, according to symptoms. The patient has steadily improved in general health,
and is apparently now on the road to recovery.

\section{THE DOMICILIARY TREATMENT OF PULMONARY TUBERCULOSIS.}

G. H. DART, M.D., M.R.C.P.Edrn., D.P.H.C.Nтав.,

TOBERCULOSIS OFFICER AND ACTING MEDICAI OFFICER OF HEALO

IN view of the fact that at the present time there is very little, if any, institutional means in most of the London boroughs for dealing with advanced cases of pulmonary tuberculosis-apart, that is, from the general hospital or infirmary - the question of how to deal with advanced cases in their homes is of the greatest importance.

London boroughs vary very much both in administrative schemes and in the character and variety of local agencies dealing with tuberculosis, but in a few respects at least the facts with regard to Greenwich may have some value for every borough.

It appears from a comparison between the number of deaths and the number of cases nursed in Greenwich that many more than half the patients who died at home did not receive skilled nursing attention.

The deaths from pulmonary tuberculosis numbered 129 during 1914 in Greenwich. Of these deaths, 50 occurred in Poor Law and similar institutions, 17 in hospital, and 62 at home. The number of cases nursed during 1914 was 46 , and 24 of these cases died during the year. There are three district nursing associations in the borough, employing in all 12 nurses.

At first sight this return, as far as regards the places where death occurred, appear's satisfactory, as more than 50 per cent. of the deaths have taken place in institutions. Also, although there were 62 deaths at lome, 46 cases had nursing supervision, with its attendant prevention of the spread of infection. It appears, however, that many of the cases nursed were not among the deaths. Satisfaction with the return is also modified by the fact that many of the patients shown as having died in an institution in reality required nursing-many of them before entering and many after leaving or before re-entering the institutions.

The leaving notifications (hospital and infirmary) during 1914 numbered 48. There were 24 deaths among theso patients. Of these deaths 5 occurred at home, and 3 of the home deaths had the benefit of nursing. The remain ing 19 deaths took place in institutions, and 2 of these patients during the period between their discharge and return were nursed.

These 19 returned patients are, of course, included in the 67 patients who died in institutions, and who, therefore, at the first glance, might be considered as not having needed nursing.

A patient who "takes his discharge" from an infirmary or hospital and becomes so rapidly worse that he dies within the year, in all probability requires home nursing whether he clies at home or enters the infirmary to dic. The large majority of institutional deaths take place in the infirmary.

These patients prefer, as a rule, to stay at home until desperately ill and leave the infirmary on the slighitest pretext.

The result of this is placed on record in the Final Report of the Departmental Committee on Tuberculosis: "Iniec tion is frequently disseminated by persons suffering from advanced pulmonary tuberculosis who are in the habit of entering and leaving Poor Law institutions, having regard merely to their own convenience, and not to the interests of their family or of the community in which they live."

Considering the advanced character of the disease in most cases treated in these institutions, it would probably, therefore, be nearer the mark if one considered all such cases in need of nursing after discharge. Certainly, if, as in Greenwich in 1914, 50 per cent. of these cases die during the same year, it is probable that the majority of the survivors have been during the year in need of attention.

If the 48 leaving notifications, minus the 5 patients that died at home, are added to the total 62 deaths at home 81 per cent. of the total deaths will be found to hare required nursing during 1914

'The greatest number of visits paid to a patient was 60 . The average, obtained from the total numbers of patients and visits, worked out at 20 visits a patient.

The work cannot be carried out by dispensary nurses anless the number attached to the dispensaries is greatly increased. If for the purpose of estimating the number: o visits required one takes, not the 81 per cent. mentioned above, but ouly 66 per cent., or, say, two-thirds of the deaths, at an average rate of 20 visits to each patient, the estimated number of visits would in many instances approach the number of visits actually paid by the nursing staff. As an illustration :

\begin{tabular}{|c|c|c|c|c|c|c|c|c|}
\hline & & \multicolumn{4}{|c|}{ Deaths. } & \multirow{2}{*}{ N. } & \multicolumn{2}{|c|}{ visits. } \\
\hline & & Total. & P. L. I. & H. & D. & & C. F. & A. \\
\hline Paddington ... & $\ldots$ & 156 & 42 & 35 & 79 & 664 & 2,925 & 2,080 \\
\hline St. Marylebone & ... & 149 & 87 & 15 & 47 & 526 & 2,219 & 1,986 \\
\hline Stepney $\quad \ldots$ & $\ldots$ & 447 & 198 & 55 & 194 & 2,038 & 10,016 & 5,960 \\
\hline
\end{tabular}

Total $=$ Total deaths 1913. P. I. I. $=$ Deaths in Poor Law institutions. H. = Deaths in hospital. D. $=$ Deaths at home. N. = Primary pulmonary notifeations. C. F. = Central Fund report visits peid. A. = Estimated visits to advanced cases.

To attempt the nursing from the dispensary of those who died at home, even if such a small number of visits as to average 20 per patient were paid, would in itself be sufficient to cripple the work in connexion with other than advanced cases.

The Greenwich Dispensary had not completed a full year in 1913. Throughout 1914 the nurses did not attempt any home nursing, even of bed-ridden patients who had formerly been dispensary patients.

$$
\begin{aligned}
& \text { Greenwich, } 1914 . \\
& \text { Deaths ... } \\
& \text { Primary pulmonary notifications } \\
& \text { Visits paid }
\end{aligned}
$$

The deaths alone, moreover, give no indication of the 
number of patients who survive for a more lengthy period, and who also require nursing during rellapses.

If to the 62 home deaths were added the 22 nursed cases who survived the year and the 38 "leaving notifications," after subtracting the deaths at home and the number nursed from the total "leaving notification," the estimated number of visits to 122 cases would be 2,440. Perhaps if this number were doubled it might indicate, as far as regards last year's figures, the amount of work required in endeavouring to prevent the spread of infection by these cases.

At least two additional whole-time nurses would be needed at Greenwich if this work were to be attempted from the dispensary. The Greenwich Dispensary nurses visit all notified patients of every description, and the district nurses are informed of those cases requiring home nursing. The dispensary nurses were formerly Ranyard nurses, and are in close touch with the Ranyard Association.

In spite of this, horverer, it is evident that a considerable number of cases do not receive adequate attention, and many no attention at all. Some of the patients move out of the district of the particular nurse who has been in attendance. Many patients who receive nursing aid recover to some extent and ask the nurses to cease calling; a later relapse is followed by a call for the doctor, but not for the nurse; and, from the point of view of prevention, careful nursing of these cases is of much greater impor. tance than medical aid. Careful nursing, backed up by medical authority, is essential. Both for the sake of the patient and of the community domiciliary treatment should always include skilled nursing in addition to medical advice. Many other patients refuse help from the nurses, or other members of the family object.

A closer connexion between the nursing associations and the public health administration might obviate some of these difficulties, and arrangements to this end are now under consideration in Greenwich. Perhaps the most desirable arrangement would be that the actual nursing of a patient receiving domicjliary treatment should be carried out by a nurse from the dispensary appointed to deal with advanced cases, and that a lealth visitor should keep the case under observation when actual nursing was not required, all, of course, under medical supervision.

Since it is generally accepted that "there can be no manner of doubt that the far advanced or dying cases constitute the greatest source of infection," it follows that any scheme for the prevention of tuberculosis which does not deal adequately with these cases will fail in its object.

\section{fftumarattor:}

\section{MEDICAL, SURGICAL, OBSTETRICAL.}

\section{WOUND DRESSING.}

Shovld a wound arrive in a very foul condition it should not be covered with a dressing directly applied. A patty pan, having a hole about the size of a shilling punched through its centre, should first be placed over the wound. Patty pans cost $2 \mathrm{~d}$. or $3 \mathrm{~d}$. a dozen at the ironmonger's, and can be bent by hand to the shape of the part. They have iolled-orer edges, and lie smoothly and comfortably on the sound skin renicte from the wound. Dressings are applied outside the pans. Nothing, therefore, touches or blocks the wound itself, and discharges freely flow into the hollow of the pan and its covering dressings. When desirable the pan is held in place by one or two strips of strapping passing across its edges. All the discharge will be found in the hollow of the pan and the dressings, and practically none in the burrows of the wound, at the subsequent dressing. The pan can be boiled and used again.

A wound should be syringed out from the bottom if it extends deeply. To this end the ordinary syringe nozzle should be replaced by 6 in. of thin copper tubing, say $\frac{1}{8}$ in. in diameter. For convenience the tube should be bent ncar its screw attachment through an angle of about 60 degrees and its free end rounded with a little solder.

For syringing, as a general rule, hypochlorous acid, the 21 rer cent. solution of Professor Lorrain Smith and his ccadjutors (cusol), should be used, and the wound dressed by applying a pad of gauze moistened with eusol, and folded to the. size of the wound treated. This is covered by a similar pad of wet wool, which holds liquid better. Over this should be placed a piece of waterproof tissue, slightly larger than the dressings beneath. Finally, a good pad of cotton-wool should be applied, extending well beyond the waterproof tissue, especially below it in the line of drainage. The size of the waterproof tissue is not unimportant as it determines that of the wool which has to project beyond it sufficiently far to absorb any discharges escaping from boneath the impervious material. In war especially economy is a virtue, and the effort to attain it encourages attention to detail. Thus treated, a wound should be clean and healthy in two or three days, if free from any foreign body or dead bone.

The best waterproof tissue procurable can be made by any one for 6d. a square yard, about one-fourth the usual price. I buy a length-say 30 yards-of "madapolin" (a thin strong calico) 42 in. wide, at $4 \frac{1}{2} \mathrm{~d}$. a yard. I tear it into yard lengths, and clip a piece at a time into cold boiled linseed oil. This I wring out by hand, and wipe off any surplus oil from the twisted mass and my hands with the yard of dry madapolin next to be treated. 'The oil-soaked piece is hung up on a horizontal wire by three safety pins, and the following day is hung the reverse way up. A second reverse is generally desirable. The whole dries in a week in an epen shed, or in about ten days in a garage in winter.

The hospital of 200 beds with which I am connected uses little else to cover wet dressings or protect splints since I introduced it. The same piece is commonly used repeatedly, as it stands scrubbing well.

Topsham, Devon.

D. W. Samways, M.D., D.Sc., M.R.C.P.

\section{SODICM SULPHATE IN DYSENTERY AND INFANTILE DIARRHOEA.}

In the Journal of November 13th Dr. Penfold refers to the discovery of the $B$. dysenteriae (Flexner) in two cases of infantile diarrhoea under his care and to its recognition by others in similar cases. I have for several years regarded infantile diarrhoea as so closely allied to pure bacillary dysentery in children that $I$ have invariably submitted them to the same treatment.

This consists, briefly, in the administration of sodium sulphate in doses, for children under 1 year, of 5 to 15 grains every two or three hours. A preliminary dose of castor oil is valuable in all cases. Milk is generally excluded from the diet for a few days. Under this treatment the mortality from this disease in my own practice has been reduced to an almost negligible percentage.

In the cases of dysentery occurring amongst the Mediterranean Forces, failure to find any one special bacillus as a causative agent appears to have been frequent in laboratories, owing probably to the bacteriological examination being made after the disease has existed for some time, as the dysentery bacilli very quickly aro crowded out by other intestinal species. Experience of bacillary dysentery in the tropics has convinced me that the administration of bismuth and opium should be aroided, and that the treatment by 1-drachm doses of sodium sulphate every hour or two is to be preferred to every other. The frequency of dosage is modified as the pain and tenesmus lessen, and the character of the stools, as shown by the disappearance of blood and mucus, im proves. The drug should be continued for some time after the stools have become entirely free from a trace of blood or mucus. No harm, but rather good, will result from the patient having three or four loose stools daily.

Both amoebic and bacillary dysentery respond at once to this treatment, but should amoebae be found later in the stools emetine should, of course, be administered. It is unnecessary otherwise. The earlier the treatment is adopted the more quickly and completely patients will recover.

W. J. J. Arvold, B.A., M.B., D.P.H.

The Royal Dental Hospital, Leicester Square, has received a donation of $£ 2,000$ from the trustees of the late Miss Harker-Smith. The Chelsea Hospital for Women has received a legacy of $£ 1,000$ bequeathed by the late $\mathrm{Mr}$. Jbhn Samuel White. 